\title{
VALORES Y CARACTERISTICAS DE PERSONALIDAD EN ESTUDIANTES DE TRABAJO SOCIAL DE LA COMUNIDAD VALENCIANA
}

JORGE GARCES FERRER, M. ${ }^{a}$ JOSE LAHOZ CAMPOS, FCO. JOSE RODENAS RIGLA Y ESTRELLA DURA FERRANDIS

Profesores SS.SS en la Escuela de Trabajo S. Universidad de Valencia

\section{SUMARIO}

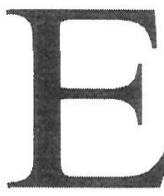

n el estudio entramos a delimitar el perfil psicosocial de los futuros trabajadores sociales en la Comunidad Valenciana. Para ello se analizan las características de personalidad y los valores (utilizando los cuestionarios CEP y SIV) de una muestra compuesta por los alumnos de 1 er $^{\text {er }}$ curso de las EUTS de Valencia y Alicante. Nuestras conclusiones posibilitan aclarar aspectos axiológicos y metodológicos relacionados con el Trabajo Social.

\section{INTRODUCCION}

El presente trabajo pretende analizar las características de personalidad de una muestra de estudiantes de Trabajo Social de dos escuelas, las de Valencia y Alicante, con el fin de delimitar el perfil psicosocial de los trabajadores sociales, y analizar cómo influirán la presencia o ausencia de determinados valores y características de personalidad en el ejercicio profesional posterior.

Los objetivos de la investigación se concretan fundamentalmente en analizar esas variables de personalidad y valores de los futuros trabajadores sociales de la Comunidad Valenciana, por un lado; por otro, averiguar la existencia de diferencias significativas entre los estudiantes de Trabajo Social de Valencia y Alicante y respecto a las variables mencionadas.

\section{Hipótesis}

Si partimos del supuesto de que el Trabajo Social abarca funciones de ayuda, promoción y educación de individuos, grupos o comunida- 
des, se podría intuir que los trabajadores sociales tendrían hipotéticamente (deseablemente) las siguientes características:

- Un grado de extroversión suficiente como para poder establecer una buena comunicación y relación social con los usuarios/as.

- Un nivel de estabilidad emocional y autocontrol necesario para tener y utilizar criterios ante las posibles decisiones sobre los recursos a utilizar.

- Suficiente grado de sinceridad frente al usuario, para no crear falsas expectativas.

- Poca capacidad de sumisión, por ser ésta incompatible con la lucha por conseguir recursos para satisfacer al máximo las demandas que siempre estarán en relación inversa a la recursos existentes.

- Capacidad de autocrítica, necesaria para alcanzar una cierta madurez tanto a nivel individual como a nivel de trabajo en equipo.

- Dada la relación personal que se establece con los usuarios y con los compañeros de trabajo, debe valorar suficientemente el apoyo, la amabilidad y la consideración en las relaciones personales.

- Lo suficientemente independientes como para tomar decisiones según sus propios criterios profesionales, proyectando dicho valor instrumental sobre la usuaria/o.

Estas y otras características conformarían, en principio, un perfil psicosocial idóneo (ideal) para el ejercicio de la profesión de Trabajo Social, pues se trata de «una profesión dedicada y comprometida a efectuar cambios sociales en general y en las formas individuales de desarrollo dentro de la misma» ${ }^{1}$. A través de nuestra investigación intentaremos comprobar si estas hipótesis se adecuan a las características reales de los futuros trabajadores sociales.

\section{METODOLOGIA}

\section{Descripción de la muestra}

El universo de este estudio lo componían los estudiantes de primer curso de las escuelas de Trabajo Social de Valencia y Alicante; la muestra fue recogida durante el curso académico 1990/91.

La muestra estaba compuesta de 169 sujetos, $86(50,9 \%)$ de Valencia y $83(49,1 \%)$ de Alicante (Ver TABLA I). Del total de alum-

${ }^{1}$ FUNDACION UNIVERSIDAD-EMPRESA: Los estudios de Trabajo Social, Col. Monografías profesionales, núm. 51, Madrid, 1983, pág. 11. 
nos, un $51,5 \%$ (27) pertenecían al grupo de mañana y al grupo de la tarde $48,5 \%$ (82), sin distinguir los alumnos que están matriculados en una escuela u otra. En cuanto al sexo de los encuestados, 139 eran mujeres, es decir un $82,2 \%$ de la muestra, frente a 30 hombres que representan el 17,8\% del total (TABLA II). En la EUTS de Valencia, de los 86 casos estudiados el $80,23 \%$ eran mujeres (69), y el $19,77 \%$ hombres (17). En la EUTS de Alicante del total el 84,3\% eran mujeres (70) y el $15,7 \%$ hombres (13).

En cuanto a las edades medias de los grupos analizados, la de Valencia es de 20,6 años y la de Alicante 20,86. Por último, respecto al estado civil de los sujetos encuestados del total de 169,157 son solteros, es decir el $92,9 \%$, y son casados representando el $4,1 \%$ y 1 sujeto separado $(0,6 \%)$.

TABLAS REFERIDAS A LA DESCRIPCION DE LA MUESTRA TABLA I

\begin{tabular}{lcc}
\hline $\begin{array}{c}\text { Número } \\
\text { Casos }\end{array}$ & $\begin{array}{c}\text { Valor } \\
\text { Absoluto }\end{array}$ & $\begin{array}{c}\text { Valor } \\
\text { Relativo }\end{array}$ \\
\hline Valencia & 86 & $50,9 \%$ \\
\hline Alicante & 83 & $49,1 \%$ \\
\hline Total & 169 & $100 \%$ \\
\hline
\end{tabular}

TABLA II

\begin{tabular}{lcc}
\hline Sexo & $\begin{array}{c}\text { Valor } \\
\text { Absoluto }\end{array}$ & $\begin{array}{c}\text { Valor } \\
\text { Relativo }\end{array}$ \\
\hline Hombres & 30 & $17,8 \%$ \\
\hline Mujeres & 139 & $82,2 \%$ \\
\hline Total & 169 & $100 \%$ \\
\hline
\end{tabular}


TABLA III

\begin{tabular}{|c|c|c|c|c|c|c|}
\hline $\begin{array}{c}\text { Estado } \\
\text { Civil }\end{array}$ & \multicolumn{2}{|c|}{$\begin{array}{l}\text { Valencia } \\
\text { Abs. Rel. }\end{array}$} & \multicolumn{2}{|c|}{$\begin{array}{c}\text { Alicante } \\
\text { Abs. Rel. }\end{array}$} & \multicolumn{2}{|c|}{$\begin{array}{c}\text { Totales } \\
\text { Abs. Rel. }\end{array}$} \\
\hline Soltero & 80 & $\begin{array}{l}93,02 \% \\
51 \%\end{array}$ & 77 & $\begin{array}{l}92,78 \% \\
49 \%\end{array}$ & 157 & $92,9 \%$ \\
\hline Casado & 4 & $\begin{array}{r}4,6 \% \\
57,1 \%\end{array}$ & 3 & $\begin{array}{c}3,61 \% \\
42,9 \%\end{array}$ & 7 & $4,1 \%$ \\
\hline Viudo & 0 & & 0 & & 0 & $0 \%$ \\
\hline Separa. & 1 & $\begin{array}{l}1,19 \% \\
100 \%\end{array}$ & 0 & & 1 & $0,6 \%$ \\
\hline Divorc. & 0 & & 0 & & 0 & $0 \%$ \\
\hline $\begin{array}{l}\text { No } \\
\text { Consta }\end{array}$ & 1 & $\begin{array}{c}1,19 \% \\
25 \%\end{array}$ & 3 & $\begin{array}{l}3,61 \% \\
75 \%\end{array}$ & 4 & $2,4 \%$ \\
\hline Totales & 86 & $50,9 \%$ & 83 & $49,1 \%$ & 169 & $100 \%$ \\
\hline
\end{tabular}

\section{Descripción de instrumentos}

Se han utilizado dos test o pruebas para delimitar el perfil psicosocial de la muestra. Para la medición de las características de personalidad, se utilizó el Cuestionario de Personalidad CEP de J. L. Pinillos, compuesto por cinco factores:

$C=$ Control: Que hace referencia a la estabilidad emocional de los sujetos, es decir, al hecho de experimentar o no cambios emocionales sin causa aparente o por razones nimias; estos cambios son de naturaleza eminentemente depresiva. No se trata de un factor de índole volitiva sino de variaciones más profundas de la emotividad.

$E=$ Extraversión: En esta escala predomina el optimismo y sociabilidad, gusto y facilidad por las relaciones sociales. Se mide la extraversión social, cuyas características principales son: la animación de carácter, facilidad y gusto por contactos sociales y relaciones, locuacidad y habilidad para dirigir reuniones. En segundo término aparecen cualidades como despreocupación y preferencia de la acción sobre la reflexión.

$P=$ Paranoidismo: Esta escala queda definida por tres características: agresividad, suspicacia y tenacidad rígida; tras ellas parece vislumbrarse una cierta convicción de la propia superioridad sobre los demás.

$S=$ Sinceridad: Esta escala intenta medir el grado de sinceridad de 
los sujetos utilizando para ello preguntas relativas a pequeñas faltas morales o sociales frecuentes en casi todo el mundo, pero cuya confesión pública o en cuestionario que va a ser leído puede resultar molesta e inducir a mentir. No obstante, no queda claro si lo que se mide realmente es la sinceridad o la auténtica asimilación de las normas sociales.

$i=$ Número de dudas: Esta escala mide la seguridad y la decisión que posee el sujeto a la hora de elegir.

En el estudio de los valores sociales e interpersonales, se utilizó el Cuestionario SIV de L. V. Gordon que contiene seis factores:

Estímulo: La subescala estímulo permite medir el grado en que los individuos prefieren ser tratados con amabilidad y comprensión por los demás, recibiendo su apoyo.

Conformismo: Esta escala revela la conformidad hacia las normas sociales, seguir estrictamente las pautas, rechazando todo aquello que no es aceptado por la sociedad, es decir ser conformista.

Reconocimiento: Esta escala consiste en ser bien visto y admirado, ser considerado como personas importantes, llamar favorablemente la atención, conseguir el reconocimiento de los demás.

Independencia: Revela el derecho a hacer lo que uno quiere hacer, ser libre para decidir por sí mismo, ser capaz de actuar según el propio criterio.

Benevolencia: Determina la predisposición a hacer cosas por los demás y compartirlas con ellos, ayudar a los poco afortunados y ser generosos.

Liderazgo: Hace referencia a la predisposición para estar al cargo de otras personas, teniendo autoridad sobre ellas, estar en un puesto de mando o poder.

\section{DESCRIPCION E INTERPRETACION DE RESULTADOS}

\section{A. Análisis descriptivo}

En este punto se desarrolla un análisis descriptivo de los datos obtenidos tras el pase de las dos pruebas consideradas necesarias para descubrir el perfil psicosocial del trabajador social (SIV y CEP). Cada prueba aparece interpretada en sus diferentes factores a través de la comparación de las puntuaciones medias obtenidas en cada factor por los sujetos muestreados, con la media y la desviación típica del estadístico de cada test, teniendo en cuenta el sexo. 


\section{III.1. Características de personalidad}

Como mencionábamos anteriormente el cuestionario de personalidad CEP está constituido por cinco escalas (Control, Extraversión, Paranoidismo, Sinceridad y Número de Dudas), pero en este artículo centraremos nuestra atención en las tres primeras.

Los datos obtenidos en la escala de Control para los alumnos de Valencia son en varones $\bar{X}=11,17$ y en mujeres $\bar{X}=9,6$; siendo la media normativa para varones 11,89 con una $\mathrm{DT}=5,72$, mientras que para mujeres $\bar{X}=10,35$ con una $\mathrm{DT}=5,21$. Comparando los resultados con las medias estándar vemos que tanto hombres como mujeres se encuentran ligeramente por debajo de estas últimas, aunque dentro de los límites inferiores de su grupo.

Respecto al grupo de Alicante la $\bar{X}$ en varones es de 9,85 y en mujeres 8,76 , situándose ambas por debajo de las medias estándar, pero dentro de los límites inferiores que marca la DT para cada sexo. Los datos obtenidos indican que los estudiantes de Trabajo Social son emocionalmente estables. Aunque las medias resultantes estén ligeramente por debajo de las medias estándar, no lo están lo suficiente como para hablar de una tendencia hacia la depresión en los futuros trabajadores sociales.

En la escala de Extraversión observamos que en el grupo de alumnos de Valencia la $\bar{X}$ en varones ha sido de 22,88 y en mujeres 23,44 ; la $\bar{X}$ estandar para varones es 20,92 (DT 7,8$)$ y 19,56 (DT 8,12) para mujeres. Al comparar se observa cómo los dos sexos superan la media general pero caen dentro del límite de la DT. En cuanto a los alumnos de Alicante, encontramos una $\bar{X}$ en varones de 25 y 23,4 en mujeres, es decir, se supera la media al igual que el grupo de Valencia; sin embargo, este grupo se acerca más al límite superior marcado por la DT.

Deducimos en general que los alumnos de primer curso de Trabajo Social muestran una actitud abierta hacia las relaciones sociales, lo cual resulta muy positivo ya que la profesión de Trabajador Social implica un contacto directo y continuo con los «usuarios/as» que acuden a las distintas instituciones y organizaciones de Servicios Sociales. Es más, ello debería elevarse a axioma metodológico en la profesionalidad del Trabajador social, y de hecho así se recoge en su código deontológico, a saber: «todos los usuarios han de ser atendidos con el mismo interés, dedicación y honestidad profesional sin excepción», «toda la tarea profesional está al servicio de la persona y se ha de dejar la relación con el cliente cuando ya no sea necesaria», o «el secreto profesional es un valor y una garantía para la profesión» ${ }^{2}$.

${ }^{2}$ COLEGIO PROFESIONAL DE DIPLOMADOS EN TRABAJO SOCIAL Y ASISTENTES SOCIALES DE CATALUNYA: "Código de Etica de los Asistentes Sociales. Revista de Servicios Sociales y Política Social, 1989, núm. 16. 
La X para el grupo de Valencia en la escala de Paranoidismo ha sido $13,64 \%$ en varones y 16,72 en mujeres, mientras que la $\bar{X}$ del grupo de referencia en varones es de 19,18 (DT 6,13) y 17,92 (DT 5,84) en mujeres. Como puede apreciarse, ambos sexos se encuentran por debajo de la media general.

En cuanto a Alicante, la X observada para varones es de 18,62 y para mujeres 17,43, ambos están ligeramente por debajo de la media normativa. Ahora bien, es de destacar la diferencia de $\overline{\mathrm{X}}$ entre los grupos. Los varones de primer curso de Trabajo Social de Valencia tienen una tendencia al paranoidismo menor que los alumnos de Alicante, con una diferencia de alrededor de cinco puntos.

Los grupos estudiados se encuentran por debajo de la media general, por lo que puede interpretarse que son menos suspicaces, agresivos y tenaces que su grupo de referencia. Lo cual nos lleva a pensar que no poseen una mentalidad autoritaria, puesto que el paranoidismo es un factor importante para medir características de personalidad intolerante, tal y como se refleja en la Escala F de Adorno. Lo anterior es importante para la labor profesional en el ámbito del Trabajo Social, donde la autodeterminación del usuario es uno de los principios inspiradores del mismo, lo cual sería incompatible con una mentalidad poco flexible por parte de nuestros profesionales.

TABLAS DEL TETS CEP

TABLA ALUMNOS EUTS VALENCIA

\begin{tabular}{lcc}
\hline $\begin{array}{c}\text { Escalas } \\
\text { CEP }\end{array}$ & $\begin{array}{c}\text { Medias } \\
\text { HOMBRES }\end{array}$ & $\begin{array}{c}\text { Medias } \\
\text { MUJERES }\end{array}$ \\
\hline Control & 11,17 & 9,6 \\
\hline Extraversión & 22,88 & 23,44 \\
\hline Paranoidismo & 13,64 & 16,72 \\
\hline
\end{tabular}

TABLA ALUMNOS EUTS ALICANTE

\begin{tabular}{lcc}
\multicolumn{1}{c}{$\begin{array}{c}\text { Escalas } \\
\text { CEP }\end{array}$} & $\begin{array}{c}\text { Medias } \\
\text { HOMBRES }\end{array}$ & $\begin{array}{c}\text { Medias } \\
\text { MUJERES }\end{array}$ \\
\hline Control & 9,85 & 8,76 \\
\hline Extraversión & 25 & 23,4 \\
\hline Paranoidismo & 18,62 & 17,43 \\
\hline
\end{tabular}




\section{A.2. Valores}

Hemos comparado los resultados obtenidos en nuestra muestra con el baremo español de estudiantes universitarios del cuestionario de L. V. Gordon (SIV); abordaremos en este punto una descripción de los datos obtenidos y una interpretación de los mismos.

En cuanto a la subescala Estímulo para el grupo de Valencia la $\bar{X}$ obtenida para varones es de 20,29 , (la $\bar{X}$ normativa es de 14,9 y la DT $4,85)$ y en el caso de las mujeres la $\bar{X}$ del grupo estudiado es de 19,02 , (la media normativa es de 14,46 , con una desviación típica de 4,59 ), con lo cual, ambos sexos se encuentran por encima de la media, superando el límite superior que marca cada desviación típica. En el caso del grupo de Alicante se obtiene una media de 18,46 para los varones y 16,5 para las mujeres, ambas medias se sitúan por encima de su $\bar{X}$ estándar respectiva, sin sobrepasar el límite superior; observamos cómo las $\tilde{\mathrm{X}}$ para los alumnos de Valencia son más elevadas que las del grupo de Alicante.

Según estos datos podemos considerar que el futuro trabajador social será una persona que gusta ser tratada con consideración y afabilidad. Este deseo puede ser extensivo al trato que el trabajador social dispensa y proyecta hacia los individuos, grupos y comunidades con los que trabaja, trato que iría encaminado a aumentar la comunicación y empatía con los clientes y su participación, indispensable para dar soluciones conjuntas a las distintas problemáticas.

Por lo que respecta al valor de Conformismo, para Valencia la $\overline{\mathrm{X}}$ obtenida en esta subescala en varones es de 12 y en mujeres de 12,28 ; las medias del grupo de referencia son de 16,49 , (DT 5,61) y 17,94 y (4,27 DT), respectivamente. En ambos casos se sitúan por debajo de la media; sin embargo, los varones se encuentran dentro del límite inferior que marca la DT 10,38, mientras que las mujeres se sitúan por debajo del límite inferior de la DT que en este caso es de 13,67. Respecto al grupo de Alicante, en cuanto a los varones la $\bar{X}$ es de 11,46 y la de las mujeres de 13,24 , en los dos casos se sitúan por debajo de la media general para cada sexo. El anticonformismo se erige aquí, pues, como valor central en la axiología de aquellos que estudian Trabajo Social; el no conformismo correlaciona genéricamente con una cierta actitud reivindicativa del futuro trabajador social, luchando por llevar a cabo cambios sociales e individuales que posibiliten y favorezcan la auto-realización y el bienestar de los individuos, grupos y comunidades. También conviene relacionar esta actitud con el rol que el/la profesional adopte frente a la institución u organización de Servicios Sociales para la cual trabaje, donde deberá compaginar la filosofía de aquella con los principios éticos inherentes del Trabajo Social.

La escala de Reconocimiento consiste en ser bien visto y admirado, conseguir el reconocimiento de los demás; aquí las $\overline{\mathrm{X}}$ para Valencia 
son 6,5 en varones y 7,97 en mujeres, mientras que la media general para los alumnos universitarios es de 7,66 con 4,09 de DT, y de 7,31 y 3,76 de DT, respectivamente para varones y mujeres. Como se puede apreciar, en los dos casos se encuentran dentro de los límites inferior y superior que marca la DT, pero las mujeres superan ligeramente la media y los varones se encuentran por debajo de su media general. En el grupo de alumnos de Alicante la $\bar{X}$ para varones es de 8,38 y la de mujeres 7,5 , ambas medias superan las medias normativas sin sobrepasar el límite superior que marca la DT.

Se puede constatar a partir de estos datos que los alumnos de primer curso de Trabajo Social buscan el respeto y la consideración en su labor profesional; esta búsqueda de reconocimiento profesional no debería ser explicada en términos de baja autoestima personal, sino que atenderá a factores más sociales tales como al escaso (re) conocimiento que la sociedad en general y la familia del alumno en particular tienen acerca de la profesión de Trabajo Social. Solamente desde 1983 la disciplina tiene un reconocimiento a nivel académico, universitario, en nuestro país. Por lo que la percepción social del papel que juega la profesión en nuestra sociedad no está lo suficientemente clara, o se supedita a una nebulosa de ambigüedad, aunque la profesionalidad de quienes lo ejerzan o han ejercido en el pasado esté fuera de toda duda.

En cuanto a la escala de Independencia que revela características como: tener derecho a hacer lo que uno quiere hacer, ser libre para decidir por sí mismo y ser capaz de actuar según el propio criterio, para los alumnos de la EUTS de Valencia se ha obtenido una $\bar{X}$ de 22 en varones y 21,89 en mujeres; mientras que la media de referencia es de 16,75 con DT de 6,35 para varones, y en mujeres 18,50 con una DT de 5,27 . En los dos casos están por encima de las medias generales respectivas. Del grupo de Alicante se obtiene una media de 22,7 en varones y 21,83 para mujeres.

Incuestionablemente los trabajadores sociales valoran criterios de libertad y autonomía, actitud positiva en el ámbito del Trabajo Social porque permite al profesional una cierta independencia en la toma de decisiones respecto a la entidad contratadora, publicada o privada, y además le lleva a buscar nuevas alternativas a la hora de solucionar los problemas que se le plantean. Es más, para conseguir la autonomía e independencia de los usuarios de los SS.SS, es preciso que este valor esté en la axiología personal del trabajador social, proyectándola hacia los primeros como anteriormente apuntábamos.

En lo referente a la escala de Benevolencia, los datos resultantes para el grupo de Valencia son, en varones una $\bar{X}$ de 22,5 y en mujeres 22,55 , siendo las medias normativas de 19,59 (DT 5,81) y 20,62 (DT $5,17)$ respectivamente, superando los dos sexos las medias pero no los 
límites superiores que marcan las DT. Del grupo de alumnos de Alicante se obtiene una media de 22,9 para hombres, 23,8 para mujeres.

Como se desprende de los datos obtenidos, los sujetos encuestados denotan un grado de benevolencia superior a sus grupos de referencia; encontramos, pues, en los futuros trabajadores sociales, una clara predisposición a ser tolerantes con los demás; ello conlleva también la inclinación a realizar acciones en su favor desinteresadamente.

La escala de Liderazgo hace referencia a la predisposición para estar al cargo de otras personas, teniendo autoridad sobre ellas o tener capacidad para un puesto de mando o poder. Aquí los alumnos varones de Valencia alcanzan una $\bar{X}$ de 8,75 y la de las mujeres de 6,82 . La media normativa se sitúa en 14,70 (DT 5,96 ) en varones, y en mujeres 10,82 (DT 5,76). La $\tilde{X}$ de ambos sexos es inferior a las $\bar{X}$ de los grupos de referencia, en el caso de los varones se encuentran en el límite que marca la DT $(8,74)$, las mujeres permanecen dentro del límite inferior. Respecto a los alumnos de la EUTS de Alicante, encontramos en los varones una media de 5,9 y 6,56 para las mujeres; también en este grupo los dos sexos se sitúan por debajo de la media; viendo los resultados obtenidos, destaca el hecho de que, en los dos grupos, los varones acusan una capacidad de liderazgo menor que las mujeres para ambos grupos estudiados en la Comunidad Valenciana.

Estos datos pueden ser útiles para que en el futuro, y desde las escuelas de Trabajo Social, se desarrollen estas capacidades y habilidades en los alumnos, ya que es conveniente que el futuro trabajador social sepa dirigir y coordinar pequeños grupos (p. e. grupos de apoyo social) o pueda desarrollar programas comunitarios de promoción social y personal a nivel colectivo.

TABLA I DEL TEST SIV

\begin{tabular}{lcc}
\hline Valencia & $\begin{array}{c}\text { Hombres } \\
\text { Medias }\end{array}$ & $\begin{array}{c}\text { Mujeres } \\
\text { Medias }\end{array}$ \\
\hline Estímulo & 20,29 & 19,02 \\
\hline Conformismo & 12 & 12,28 \\
\hline Reconocimiento & 6,5 & 7,97 \\
\hline Independencia & 22 & 21,89 \\
\hline Benevolencia & 22,5 & 22,55 \\
\hline Liderazgo & 8,75 & 6,82 \\
\hline
\end{tabular}




\section{TABLA II DEL TEST SIV}

\begin{tabular}{lcc}
\hline Alicante & $\begin{array}{c}\text { Hombres } \\
\text { Medias }\end{array}$ & $\begin{array}{c}\text { Mujeres } \\
\text { Medias }\end{array}$ \\
\hline Estímulo & 18,46 & 16,5 \\
\hline Conformismo & 11,46 & 13,24 \\
\hline Reconocimiento & 8,38 & 7,5 \\
\hline Independencia & 22,7 & 21,83 \\
\hline Benevolencia & 22,9 & 23,8 \\
\hline Liderazgo & 5,9 & 6,56 \\
\hline
\end{tabular}

B. Análisis de diferencias

En este apartado se pretendía averiguar si existían diferencias significativas entre los dos grupos estudiados, Valencia y Alicante, para las siguientes variables: del cuestionario CEP los factores de Control, Extraversión y Paranoidismo; del test SIV, las subescalas: estímulo, conformismo, reconocimiento, independencia, benevolencia, liderazgo.

Para llevar a cabo este análisis de diferencias entre grupos se ha aplicado estadística paramétrica univariada (Prueba T); a partir de esta prueba se observó, como muestran las tablas, que todos los resultados obtenidos son inferiores a 1,96, lo cual indica que no hay diferencias significativas entre los dos grupos de alumnos para los factores analizados; ello viene a confirmar que el perfil psicosocial de los alumnos de Valencia y Alicante es muy semejante, no habiendo diferencias significativas por lo que respecta a características de personalidad y valores.

TABLA I

\begin{tabular}{lc}
\hline \multicolumn{1}{c}{ Test CEP } & Valor de $T$ \\
\hline Control & 0,35 \\
\hline Extraversión & 1,39 \\
\hline Psico-Ticismo & 0,72 \\
\hline
\end{tabular}


TABLA II

\begin{tabular}{lc}
\hline \multicolumn{1}{c}{ Test SIV } & Valor de $T$ \\
\hline Estímulo & 1 \\
\hline Conformismo & $-0,57$ \\
\hline Reconocimiento & 0,22 \\
\hline Independencia & $-0,3$ \\
\hline Benevolencia & $-0,11$ \\
\hline Liderazgo & 0,16 \\
\hline
\end{tabular}

\section{CONCLUSIONES}

Este estudio nos ha permitido analizar las variables de personalidad y valores de los futuros trabajadores sociales de la Comunidad Valenciana. A nuestra muestra, compuesta por 169 sujetos de primer curso de Trabajo Social, de los que $82,2 \%$ eran mujeres y sólo el $17,8 \%$ varones, aplicamos los cuestionarios CEP de J. L. Pinillos y SIV de J. V. Gordon, como instrumentos para poder deducir el perfil psicosocial de estos futuros profesionales.

Nos encontramos con personas generalmente estables emocionalmente, extrovertidos, receptivos y abiertos a nuevas ideas, así como a relaciones sociales. Demuestran una actitud inconformista que bien se podría relacionar con la independencia y autonomía que debería caracterizar idealmente a un trabajador social. Buscan al reconocimiento profesional en la comunidad, debiendo llegar a un equilibrio entre sus expectativas y la realidad en que se encuentra inmerso actualmente el Trabajo Social. Todo ello confirma nuestras hipótesis iniciales de partida. No obstante y relacionado con los dos aspectos anteriores, cabe mencionar la falta de motivación o de preparación (pues estamos trabajando con muestras de primer curso) para asumir roles de liderazgo, lo cual podría perfeccionarse con adecuados programas desde las Escuelas de Trabajo Social en cursos posteriores.

Hay que decir, y siguiendo con los objetivos que se pretendian alcanzar, que no hemos encontrado diferencias significativas en cuanto a características de personalidad y valores entre los alumnos de las Escuelas de Trabajo Social de Alicante y Valencia. El presente estudio puede servir para analizar el sistema axiológico y las características de personalidad de aquellas personas que estudian actualmente en las Escuelas de Trabajo Social; ello no sólo podría ser un predictor de la efi- 
cacia en la práctica profesional sino del compromiso inicial y posterior de quienes deciden especializarse y optan por esta disciplina científica.

\section{BIBLIOGRAFIA}

Becker, H. S.: Sociological Work: Method and Substance, Aldine, Chicago, 1970.

Fink, Arthur E.: The Field of Social Work, Sage, Beverly Hills, 1985. 